\title{
Grafting from a Hybrid DNA-Covalent Polymer by the Hybridization Chain Reaction
}

\author{
Willem E. M. Noteborn, ${ }^{\dagger}$ Joeri A. J. Wondergem, ${ }^{\dagger}$ Anastasiia Iurchenko, ${ }^{\dagger}$ Farhad Chariyev-Prinz, ${ }^{\dagger}$ \\ Dominique Donato, ${ }^{\ddagger}$ Ilja K. Voets, ${ }^{\S}$ Doris Heinrich, ${ }^{\ddagger, \|}$ and Roxanne E. Kieltyka* ${ }^{* \dagger \odot}$ \\ ${ }^{\dagger}$ Department of Supramolecular and Biomaterials Chemistry, Leiden Institute of Chemistry, Leiden University, P.O. Box 9502, \\ 2300 RA Leiden, The Netherlands \\ ${ }^{\ddagger}$ Biological and Soft Matter Physics, Huygens-Kamerlingh Onnes Laboratory, Leiden University P.O. Box 9504, 2300 RA Leiden, \\ The Netherlands \\ ${ }^{\S}$ Laboratory of Physical Chemistry and Laboratory of Macromolecular and Organic Chemistry, Department of Chemical \\ Engineering and Chemistry and Institute of Complex Molecular Systems, Eindhoven University of Technology P.O. Box 513, \\ 5600 MB Eindhoven, The Netherlands \\ "Fraunhofer Institute for Silicate Research ISC, Neunerplatz 2, 97082 Würzburg, Germany
}

\section{Supporting Information}

ABSTRACT: Nucleic acid-polymer conjugates are an attractive class of materials endowed with tunable and responsive character. Herein, we exploit the dynamic character of nucleic acids in the preparation of hybrid DNA-covalent polymers with extendable grafts by the hybridization chain reaction. Addition of DNA hairpins to an initiator DNA-dextran graft copolymer resulted in the growth of the DNA grafts as evidenced by various characterization techniques over several length scales. Additionally, aggregation of the initiator DNA-graft copolymer before the hybridization chain reaction was observed resulting in the formation of kinetically trapped aggregates several hundreds of nanometers in diameter that could be disrupted by a preheating step at $60{ }^{\circ} \mathrm{C}$ prior to extension at room temperature. Materials of increasing viscosity were rapidly formed when metastable DNA hairpins were added to the initiator DNA-dextran grafted copolymer with increasing concentration of the components in the mixture. This study shows the potential for hierarchical self-assembly of DNA-grafted polymers through the hybridization chain reaction and opens the door for biomedical applications where viscosity can be used as a readout.

\section{INTRODUCTION}

Nucleic acids are powerful tools for the construction of materials because of their sequence programmability and predictable dimensions. ${ }^{1-4}$ Consequently, DNA nanotechnology has exploited their use as a structural unit for the bottom-up self-assembly of numerous discrete two and three-dimensional architectures. $^{5-8}$ More recent developments within the field have centered on taking advantage of the dynamic properties of DNA through the use of strand displacement reactions to provide reconfigurable and autonomous functions. ${ }^{9-11}$ Strand displacement is a reaction that is fuelled by the free energy released in the hybridization of partial or fully complementary DNA strands through branch migration. ${ }^{12}$ Catalyzed hairpin assembly (CHA), ${ }^{13}$ entropy-driven catalysis (EDC), ${ }^{14}$ and the hybridization chain reaction $(\mathrm{HCR})^{15,16}$ rely on strand displacement cascades to create multilayered adaptable and reconfigurable DNA-based circuits, ${ }^{17}$ autonomous DNA walkers, ${ }^{18,19}$ and amplifiers. ${ }^{20,21}$ These techniques can be useful for a range of applications from smart therapeutics to diagnostics, ${ }^{10,22,23}$ using gel electrophoresis, fluorescence, and electrochemical signals as readouts.

Beyond DNA nanotechnology, the inherent structural and dynamic features of nucleic acids can be an invaluable means to tailor the morphology and responsiveness of polymer materials in a programmable and tunable fashion. ${ }^{24-28}$ Often DNA is introduced as the water-soluble domain of a block copolymer to form responsive micellar structures and hydrogels. ${ }^{29-34}$ Although numerous reports have demonstrated the use of a block copolymer approach, graft copolymers can provide additional handles to modify the polymer architecture through variation of grafting densities, lengths and the choice of the backbone itself. ${ }^{35,36}$ The consequence of these structural modifications can result in a broader range of morphologies, such as worms, spheres, and cylinders. 37,38

Most synthetic strategies to prepare graft copolymers involve grafting from, to, and through the covalent polymer backbone to permanently fix polymeric side chains. ${ }^{38}$ A more recent development involves grafting strategies based on noncovalent interactions, opening the door to a whole new range of graft copolymer materials that can be tunable, responsive, and dynamic. ${ }^{37}$ For example, noncovalent "grafting onto" approaches through

Received: December 9, 2017

Revised: $\quad$ May 6, 2018

Published: July 5, 2018 
Scheme 1. (A) Initiator DNA-Dextran Graft Copolymer Synthesis ${ }^{a}$ and (B) Schematic Representation of HCR-Driven Graft Extension from an initiator DNA-Dextran Graft Copolymer by HP1 and HP2

A
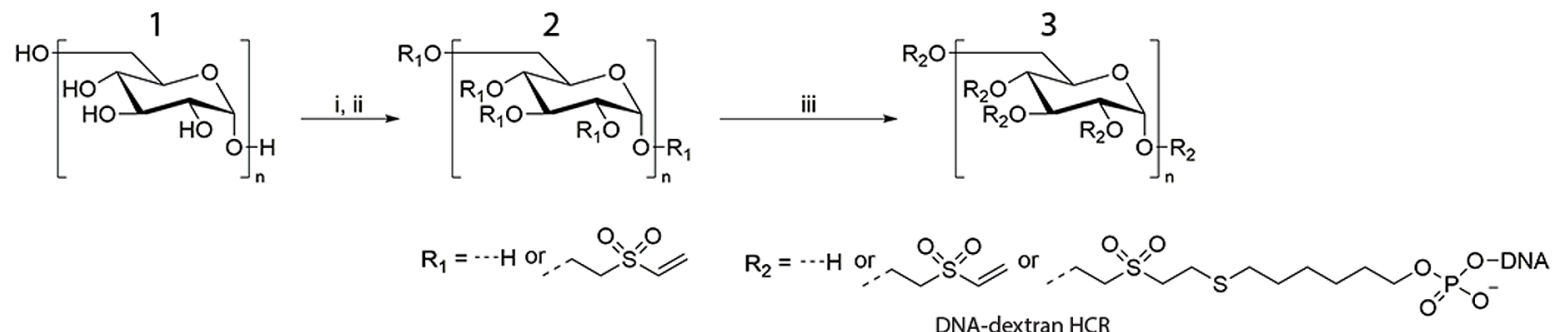

B
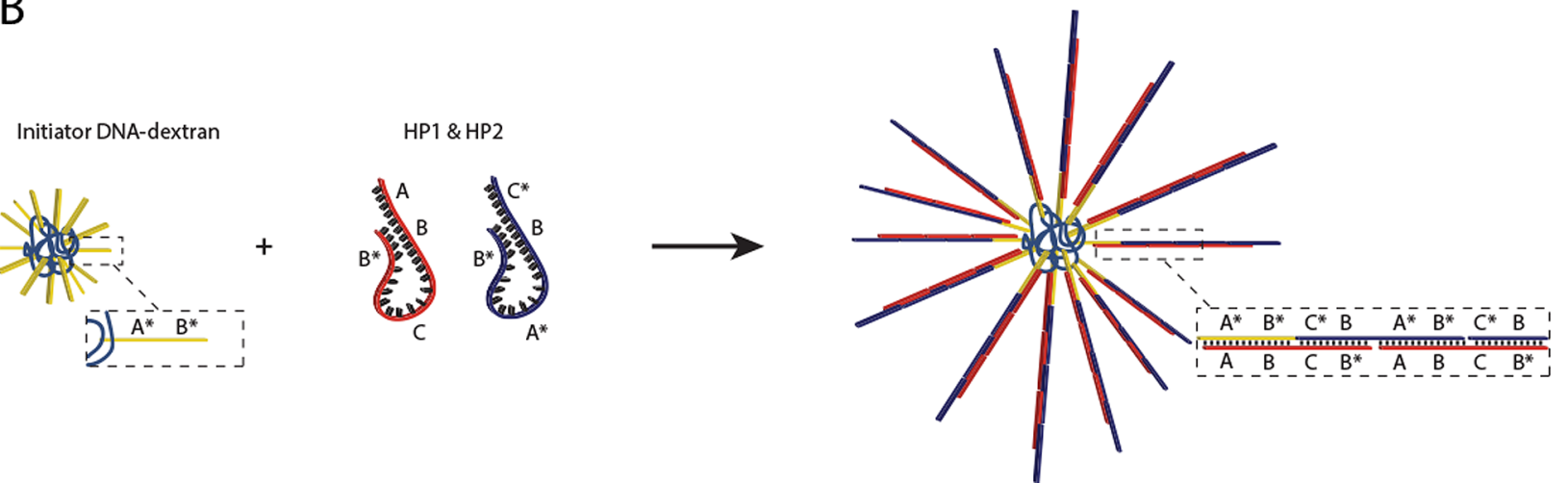

${ }^{a}$ Dextran (1) $\left(M_{\mathrm{n}}: 10 \mathrm{kDa}, \mathrm{n}_{\mathrm{av}}: 62\right)$ was reacted with divinyl sulfone yielding dextran-vinyl sulfone (dextran-VS, DS = 19) (2). Chemoselective ligation of a sulfhydryl-modified HCR DNA initiator strand by a Michael addition reaction on dextran-VS (3). Reaction conditions: (i) $0.1 \mathrm{M} \mathrm{NaOH}$, divinyl sulfone, (ii) $5 \mathrm{M} \mathrm{HCl}$, and (iii) $0.1 \mathrm{M} \mathrm{PBS} \mathrm{pH} 8.5$, using a 1:3 ratio of 5'-sulfhydryl-modified HCR initiator DNA to the vinyl sulfone groups on dextran.

molecular recognition motifs have been used to append organic molecules and biopolymers to enable structural transitions. ${ }^{39-42}$ Therefore, combining graft copolymers with dynamic DNA nanotechnology can yield a new class of grafted polymer hybrids that respond through highly specific molecular interactions in a programmable and dynamic fashion, with important consequences over several length scales.

Herein, we report the use of the hybridization chain reaction on a grafted DNA-covalent polymer to drive graft extension in a dynamic way. Autonomous growth of the nucleic acid grafts from a covalent polymer backbone is expected when supplied with two metastable hairpins (HP1 and HP2) that can undergo an energetically favorable cascade of controlled strand displacement reactions (Scheme 1). In this reaction, the DNA hairpins coexist stably in solution and are triggered when exposed to a single stranded DNA initiator complementary to a toehold on one of the hairpins. ${ }^{15}$ We herein examine DNA side chain extension on an initiator DNA graft copolymer by the hybridization chain reaction and its self-assembly from the molecular to the macroscopic scale.

\section{RESULTS AND DISCUSSION}

To synthesize the initiator DNA-grafted copolymer for triggering the hybridization chain reaction, vinyl sulfone groups were first introduced on dextran for subsequent bioconjugation with DNA. Dextran was selected as a backbone to prepare the DNA-grafted polymers because it is a naturally occurring and biocompatible FDA approved polymer that can readily be modified due to the abundance of hydroxyl groups. Dextran $\left(M_{\mathrm{n}}=10 \mathrm{kDa}\right)$ was reacted with divinyl sulfone (using 1.5 mol equiv with respect to all hydroxyl groups) under basic conditions $(0.1 \mathrm{M} \mathrm{NaOH})$ for $0.5 \mathrm{~min}$ with thorough vortexing and immediate quenching by the addition of $5 \mathrm{M} \mathrm{HCl}$ followed by dialysis purification
(75\% yield). ${ }^{43}$ By controlling the molar equivalents and reaction time, a reproducible degree of substitution of $31 \%$ (i.e., 19 hydroxyl groups distributed randomly per dextran chain) was obtained as determined by ${ }^{1} \mathrm{H}$ NMR measurements (see Supporting Information). Additionally, size exclusion chromatography (SEC) showed no change in dispersity $(\mathrm{B} \sim$ 1.05 ) or size of the vinyl sulfone substituted polymers.

In a subsequent step, dithiothreitol(DTT)-mediated deprotection of the 5 -disulfide protected initiator DNA strand was pursued to enable its conjugation to the dextran-vinyl sulfone (dextran-VS) polymer by vinyl sulfone thiol-Michael addition. Excess DTT was removed by an ethyl acetate extraction to prevent a competitive reaction with the vinyl sulfone groups on dextran and the deprotected 5'-sulfhydryl DNA. The conjugation reaction was then carried out immediately by mixing the freshly reduced 5 -sulfhydryl DNA with dextran-VS in PBS at $\mathrm{pH} 8.5$ overnight under inert conditions. The synthesized initiator DNA-dextran graft copolymer conjugates were assessed by agarose gel electrophoresis (Figure 1A, and Figure S1 for full details of initiator DNA deprotection and coupling to dextran). The unreacted DNA (lane 1, bottom), was observed as two bands, one of the deprotected sulfhydryl-DNA (bottom diffuse DNA band) and the other consisting of the two DNAs connected by a disulfide bond (sharp, middle DNA band) generated by spontaneous oxidation, in comparison to a large, slowly migrating and smeared band consistent with the formation of the initiator DNA-dextran conjugate (lane 1, top) with a variable degree of DNA substitution. ${ }^{44}$ Analysis of the agarose gels by densitometry revealed that $74 \%$ of the added 5'-sulfhydryl DNAs were conjugated to dextran, which corresponds to approximately 14 DNA grafts per dextran chain on average. Gel electroelution was used to separate and remove the unreacted initiator DNA from the initiator DNA-dextran 


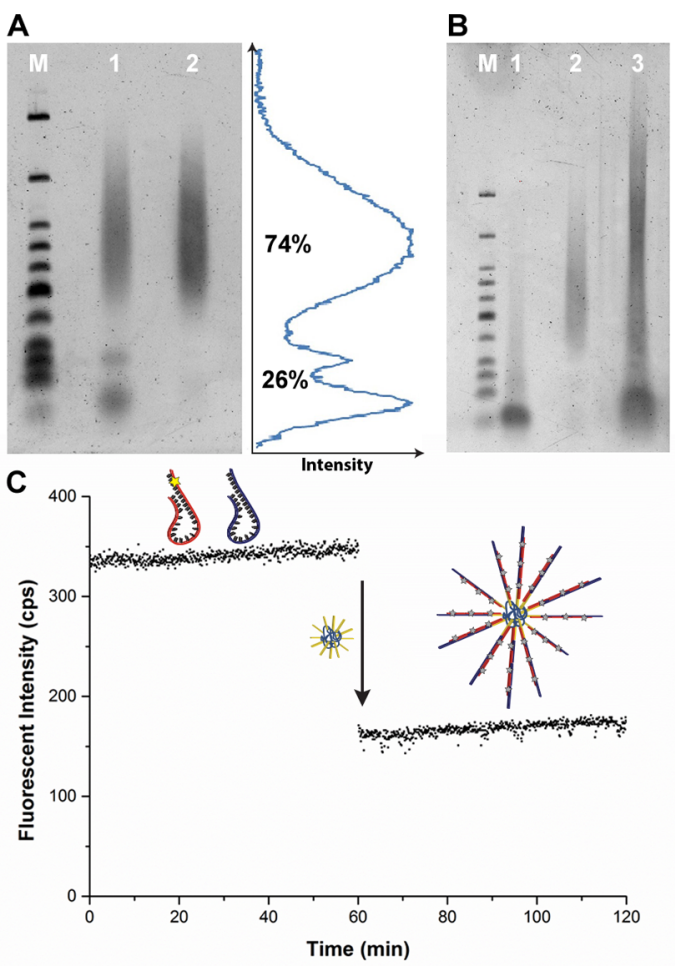

Figure 1. (A) Agarose gel electrophoresis (2\%) of the initiator DNAdextran graft copolymer before (lane 1) and after purification (lane 2). Lane $M$ contains a low molecular weight double stranded DNA ladder ranging from 25 to $766 \mathrm{bp}$. Densitometry plot shown to the right of gel. (B) Agarose gel electrophoresis (2\%) showing the products of the hybridization chain reaction on the initiator DNA-dextran graft copolymer. Combination of HP1 and HP2 (600 nM each, lane 1), initiator DNA-dextran graft copolymer (200 nM, lane 2), HCR of HP1 and HP2 on the initiator DNA-dextran graft copolymer (600 nM of each DNA hairpin and $200 \mathrm{nM}$ of initiator DNA-dextran, lane 3). Lane $\mathrm{M}$ contains a low molecular weight DNA ladder ranging from 25 to $766 \mathrm{bp}$. (C) Fluorescence time course measurement of $\operatorname{HP1}-2 \mathrm{AP}\left(\lambda_{\mathrm{ex}}=303 \mathrm{~nm}, \lambda_{\mathrm{em}}=365 \mathrm{~nm}\right)$ with HP2 $(600 \mathrm{nM}$ each $)$ for $60 \mathrm{~min}$ with subsequent addition of the DNA-dextran initiator $(200 \mathrm{nM})$ to trigger DNA side chain extension.

graft copolymer and dialysis was subsequently performed to provide a final yield of $70 \%$. Complete removal of the unreacted 5'-sulfhydryl DNA from the DNA-dextran graft copolymer was displayed on an agarose gel (2\%) (Figure 1A, Lane 2).

The capacity of the initiator DNA-dextran graft copolymer to trigger HCR was initially evaluated by gel electrophoresis and fluorescence spectroscopy on dilute solutions to provide insight into the self-assembly process at the molecular scale. Prehybridized DNA hairpins (HP1, HP2) thermally annealed in $5 \mathrm{X}$ saline-sodium citrate (SSC) buffer were mixed in equimolar quantities and added to the initiator DNA-dextran graft copolymer in the same buffer to enable the growth of the grafts. Agarose gel electrophoresis (2\%) was performed $1 \mathrm{~h}$ after the start of the reaction and showed that the addition of the folded HP1 and HP2 to the DNA-dextran graft copolymer resulted in increased retention of the polymer initiator (Figure 1B, lane 3). In contrast, lower gel retention of the negative controls including the initiator DNA-dextran graft copolymer (Figure 1B, lane 2) and the metastable hairpins only (Figure 1B, lane 1) were observed, underpinning the occurrence of the hybridization chain reaction on the dextran polymer by opening of the metastable hairpins. When compared to initiation by a DNA strand on its own, significantly higher gel retention of the initiator DNA-graft copolymer was detected (Figure S2). Moreover, the effect of increasing the ratio of HP1 and HP2 relative to the initiator DNA-graft copolymer from 1 to 9 was probed by gel electrophoresis (Figure S3). In these experiments, incomplete reaction of the hairpins during the polymerization process was observed (lane 3, lower band), which may indicate inaccessibility of some of the $\mathrm{B}^{*}$ toehold domains for extension (vide infra) due to steric effects from neighboring DNAs on the polymer backbone. The highest molecular weight products with the least unreacted hairpins were observed for an initiator DNAdextran:HP1:HP2 1:3:3 ratio. Hence, this composition was selected for subsequent experiments.

Nucleic acid fluorescence quenching experiments involving a 2-aminopurine (2-AP) functionalized hairpin 1 (HP1-2AP) were further used to support the results of the gel electrophoresis experiments. In this assay, 2-AP-labeled oligonucleotides display fluorescence in their single stranded form, but become rapidly quenched through stacking with adjacent bases when hybridized. ${ }^{15,45,46}$ The decrease in fluorescence intensity can be directly related to hairpin polymerization as previously demonstrated by the Pierce group for a DNA only system where the 2-AP label was positioned in the 5'-sticky end of HP1. ${ }^{14}$ As a control, stability of the folded HP1-2AP and HP2 hairpins on their own, in the presence of dextran-VS, and their polymerization with the addition of an initiator DNA strand were examined. Initially, a stable fluorescence signal was recorded for HP1-2AP mixed with HP2 (Figure 1C). Upon addition of either the DNA initiator strand itself (Figure S4) or the initiator DNA-dextran graft copolymer (Figure 1C), the fluorescence signal was rapidly quenched. Addition of the dextran-VS polymer did not trigger any fluorescence quenching (Figure S4). Interestingly, using either the initiator DNA-dextran graft copolymer or initiator DNA led to similar quenching behavior, suggesting that once side chain extension is started on the polymer it occurs in a similar manner.

Because of our interest in using the hybridization chain reaction to drive the formation of soft materials, we examined the morphology of the DNA-graft copolymer self-assemblies at the nanoscale by dynamic light scattering (DLS), small-angle $\mathrm{X}$-ray scattering (SAXS) and atomic force microscopy (AFM). To roughly estimate the polymer dimensions before and after DNA side chain extension, graft lengths were approximated using dimensions of typical B-form DNA and assuming a conformation of rigid rods, as the maximum graft length is likely below the persistence length $(150 \mathrm{bp})$. However, it is anticipated that the estimated graft lengths are shorter due to the existence of single strands before, and single stranded or nicked regions in the DNA grafts after hairpin addition. Thus, the diameter of the initiator DNA-dextran graft copolymer before side chain extension assuming a spherical morphology and random coil conformation of dextran $\left(M_{\mathrm{w}} \sim 10 \mathrm{kDa}\right)^{47}$ with a $4 \mathrm{~nm}$ diameter, is estimated to be approximately $16 \mathrm{~nm}$, and after polymerization using a 1:3:3 ratio of initiator DNAdextran: HP1: HP2 roughly $76 \mathrm{~nm}$.

The various components (HP1, HP2, HP1 and HP2, dextran-VS, initiator DNA-dextran, and the HCR reaction mixture) in SX SSC buffer were sized by DLS at room temperature. We found average hydrodynamic diameters of 6 and 8 $\mathrm{nm}$ for the individual hairpins and their combination, respectively. A diameter of $5 \mathrm{~nm}$ was measured for dextran-VS, which is on par with previously reported values for dextran. 47,48 
Interestingly, the initiator DNA-dextran graft copolymer appears to be far larger, exhibiting a hydrodynamic diameter of $500 \mathrm{~nm}$ that is inconsistent with the dimensions of an individual DNAgrafted copolymer (Figure 2A). Moreover, the subsequent

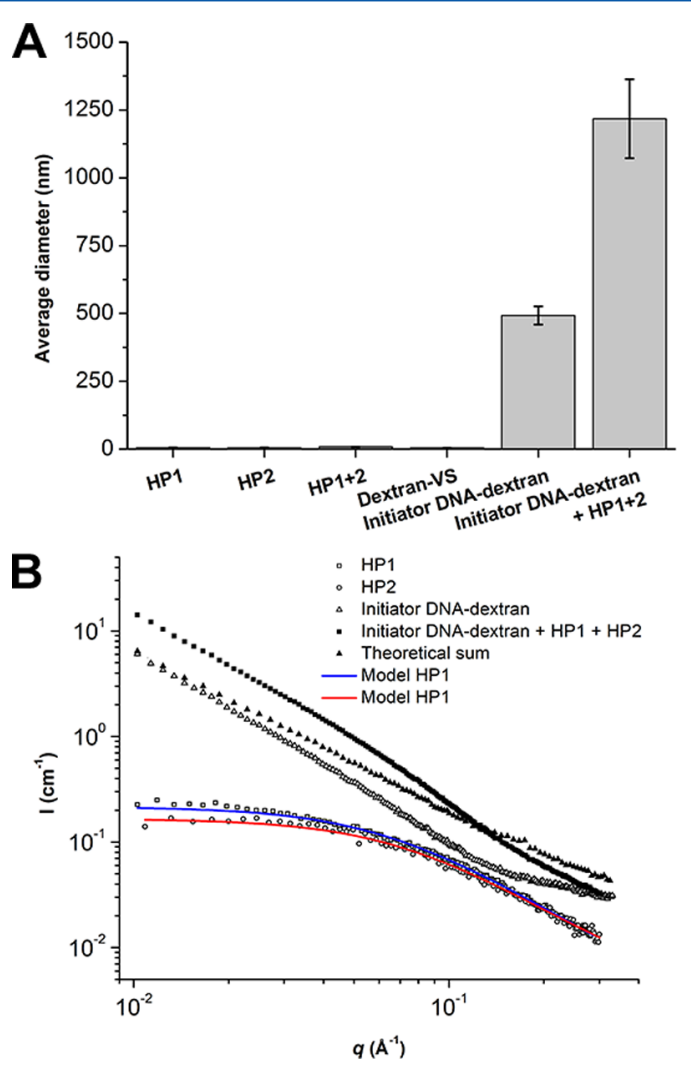

Figure 2. Hybridization chain reaction on initiator DNA-graft copolymers at room temperature. (A) Particle size distributions measured by DLS of HP1, HP2, HP1, and HP2, dextran-VS, initiator DNA-dextran graft copolymer, and the initiator DNA-dextran graft copolymer after performing hybridization chain reaction at room temperature $(N=3)$. (B) SAXS profiles of HP1 (blue) and HP2 (red) modeled with a form factor for Gaussian chains, the initiator DNAdextran before (black, open triangles) and after DNA side chain extension (black, cubes) and a theoretical summated profile of the HCR components. All samples were prepared in SX SSC buffer.

addition of HP1 and HP2 to the initiator DNA-dextran graft copolymer to facilitate graft extension resulted in the formation of micrometer-sized aggregates. The measured large diameter of the aggregates, even before addition of HP1 and $\mathrm{HP} 2$, suggests that aggregation of the initiator DNA-graft copolymer exists before and after DNA side chain extension (vide infra). In comparison, the DNA initiator on its own revealed particles sizes after hairpin addition on the order of $20 \mathrm{~nm}$, suggestive of a lack of aggregation between DNA polymers (Figure 2A).

SAXS experiments were performed to corroborate the DLS experiments in dilute solution. SAXS profiles were collected for the individual hairpins and the DNA-dextran graft copolymer before and after addition of both HP1 and HP2 at room temperature (Figure 2B). Modeling of the HP1 and HP2 SAXS profiles with a form factor for Gaussian chains yielded a radius of gyration $\left(R_{\mathrm{g}}\right)$ of $2.5 \pm 0.3 \mathrm{~nm}$ for HP1 and $2.3 \pm 0.3 \mathrm{~nm}$ for HP2. In agreement with the light scattering results, aggregates with sizes above the resolution of the instrument $\left(\pi / q_{\min }=31 \mathrm{~nm}\right)$ were observed for the initiator DNA-dextran graft copolymer
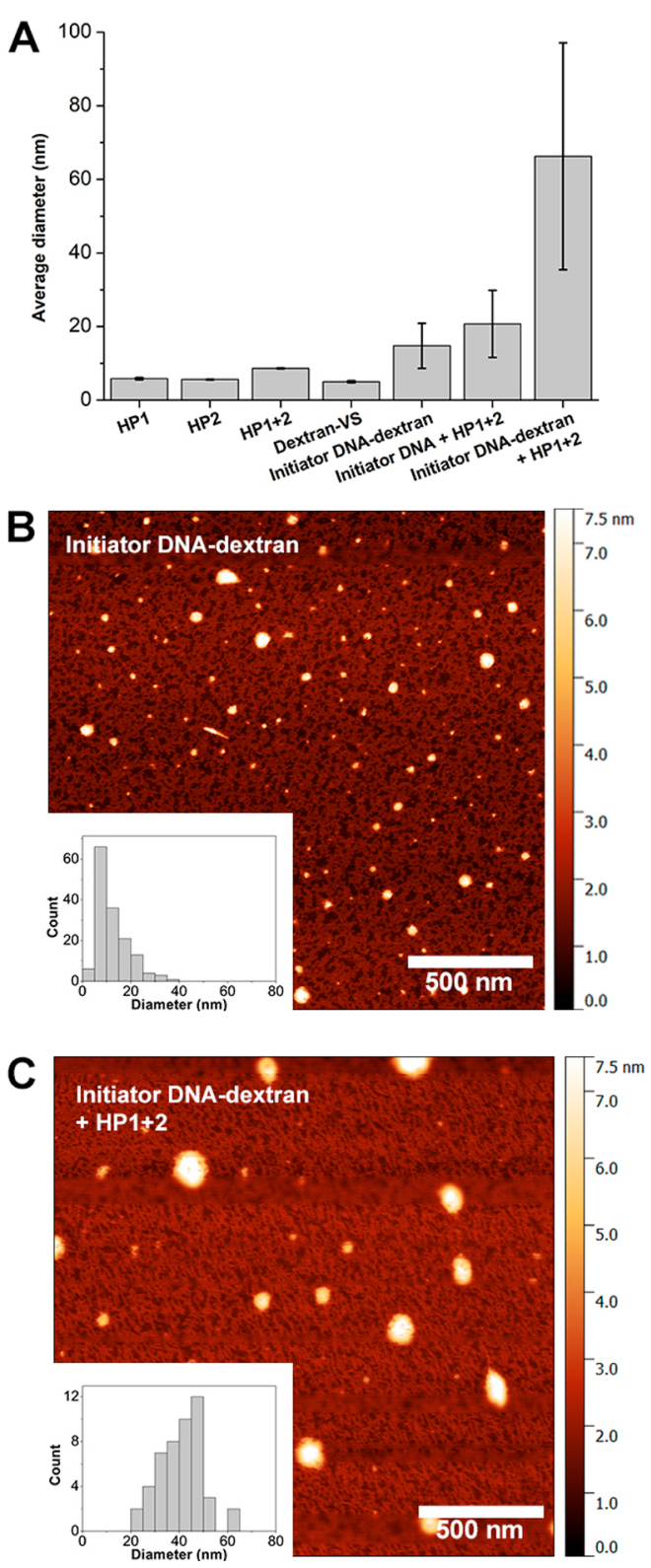

Figure 3. Hybridization chain reaction on DNA-initiator polymers preheated at $60{ }^{\circ} \mathrm{C}$ for $10 \mathrm{~min}$ and cooled to room temperature. (A) Particle size distributions measured by DLS of HP1, HP2, HP1 and HP2, dextran-VS, initiator DNA with HP1 and HP2, initiator DNA-dextran graft copolymer and the initiator DNA-dextran graft copolymer after DNA side chain extension at room temperature $(N=3)$. (B, C) Atomic force micrographs (AFM) of drop-casted samples of initiator DNA-dextran graft copolymers before (B) and after executing HCR with HP1 and HP2 (C) (below). Scale bar is $500 \mathrm{~nm}$. Insets: histograms of DNA-dextran particle diameter. All samples were prepared in 5X SSC buffer.

before and after addition of HP1 and HP2. Importantly, the experimental SAXS profile of the three-component mixture of initiator DNA-dextran graft copolymer, HP1 and HP2 is distinct from the theoretical SAXS profile computed as the sum of profiles of the individual components in the mixture (Figure 2B). This difference strongly suggests that the hairpins interact with the initiator DNA-dextran graft copolymer aggregates, triggering a conformational change when mixed.

To better follow the extension of the initiator DNA grafts on the dextran polymer, the potential for thermal disruption of the 
Scheme 2. Proposed Mechanism of Initiator DNA-Graft Copolymer Self-Assembly before and after the Hybridization Chain Reaction $^{a}$

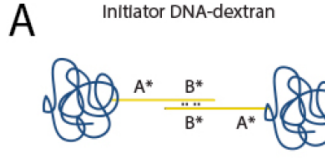

Weak homodimer interactions

B

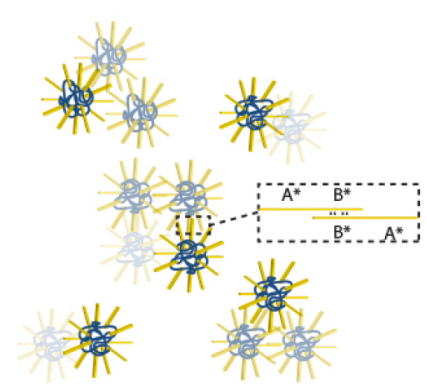

Initiator DNA-dextran

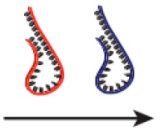

HP1 \& HP2
Growth of polymer grafts by HCR and formatlon of weak homodimers

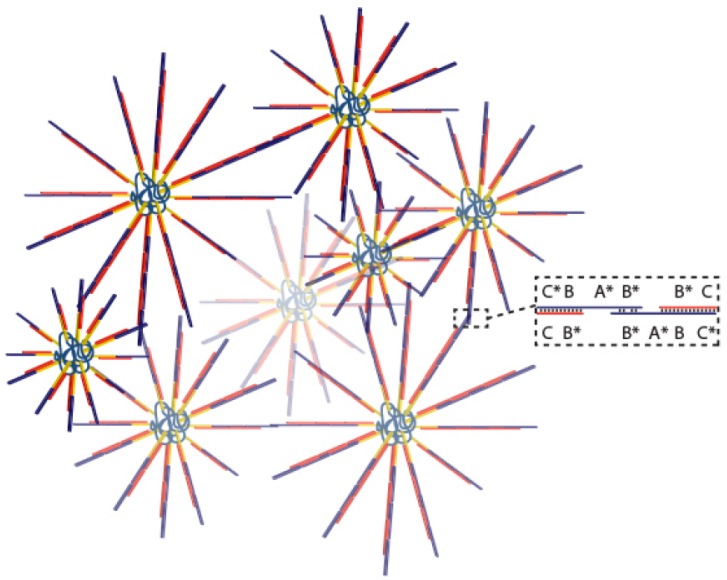

DNA-dextran HCR-based hydrogel

${ }^{a}$ (A) Initiator DNA-dextran graft copolymer bearing multiple initiator sequences can aggregate through weak homodimer interactions in domain B*. Addition of HP1 and HP2 starts the energetically favorable hybridization chain reaction on the initiator DNA-dextran graft copolymer to extend the DNA grafts. Exposed single stranded B* domains on HP1 and HP2 incorporated on the grafted supramolecular polymer can form weak homodimer interactions to form larger aggregated structures. (B) Extension of DNA grafts by the hybridization chain reaction on the initiator DNA-graft copolymers. Addition of HP1 and HP2 results in growth of the DNA grafts on the initiator DNA-graft copolymer aggregates with the potential for weak homodimer interactions facilitating secondary aggregation to occur.

aggregates was pursued prior to the hybridization chain reaction. The room temperature-prepared initiator DNA-graft copolymer solution was heated for $10 \mathrm{~min}$ at $60{ }^{\circ} \mathrm{C}$ and cooled to room temperature before the addition of $\mathrm{HP} 1$ and $\mathrm{HP} 2$, and the hydrodynamic diameter was measured by DLS at room temperature before and after hairpin addition (Figure 3A). Before hairpin addition, a particle population with a diameter of $14 \pm 6 \mathrm{~nm}$ was recorded, which increased to $66 \pm 30 \mathrm{~nm}$ after DNA extension. Interestingly, these low values contrast sharply with the far larger diameters registered without the preheating step and are comparable to the estimated diameters of the individual graft copolymers before and after HCR. These results suggest that the large sized aggregates obtained upon direct mixing at room temperature (without preheating) are kinetic products.

The changes in hydrodynamic diameter of the DNA-dextran conjugates were further supported by atomic force microscopy (AFM) measurements on the samples treated at $60{ }^{\circ} \mathrm{C}$ prior to performing HCR. The DNA-graft copolymer self-assemblies were drop-casted on mica and washed with water to remove buffer salts prior to imaging. Preheating the sample to $60{ }^{\circ} \mathrm{C}$ before DNA side chain extension on the initiator DNA-dextran graft copolymer showed small aggregates highly disperse in diameter (Figure 3B, $14 \pm 10 \mathrm{~nm}$ ). These spherical aggregates grew in size after addition of HP1 and HP2 (Figure 3C, $40 \pm$ $18 \mathrm{~nm}$ ) as observed by DLS. However, with or without heat treatment in both DLS and AFM experiments large deviations in the diameter before and after hairpin addition were recorded between samples, pointing out the influence of the dispersity of the dextran polymer, DNA conjugation and aggregation of the DNA-polymer conjugates on the observed results.
Taken together, the results of the experiments performed at room temperature (gel electrophoresis, fluorescence quenching, DLS and SAXS) or by preheating the samples prior to DNA hairpin addition (DLS and AFM) point to the initiation of the hybridization chain reaction by the initiator DNA-graft copolymer.

To understand the origin of the unexpected aggregation observed in DLS and SAXS of the DNA initiator-dextran copolymer samples prepared at room temperature, analysis of the DNA sequences used for self-assembly by NUPACK was performed. ${ }^{49}$ These investigations revealed weak homodimer interactions between four nucleotides in the initiator DNA strands $\left(\mathrm{B}^{*}\right)$, and HP1 and HP2 $\left(\mathrm{B}^{*}\right)$ once hybridized, with a computed free energy of $-11.09 \mathrm{kcal} / \mathrm{mol}$ (Scheme $2 \mathrm{~A}$ ). This value is in contrast to a computed free energy of $-40.86 \mathrm{kcal} / \mathrm{mol}$ for each formed duplex during the hybridization chain reaction. The potential for weak homodimer interactions between initiator and hairpin DNA strands may provide opportunities for both intramolecular looping within and intermolecular crosslinking between the DNA-grafted polymers to occur before and after the hybridization chain reaction as encountered in the synthesis of conventional graft copolymers. ${ }^{50}$ However, it is likely that the potential for multiple weak homodimer interactions between DNA-grafted copolymers due to their multivalent DNA presentation facilitates their secondary aggregation into large aggregates.

We further examined the potential to prepare hybrid-DNA polymer materials through the hybridization chain reaction by particle-tracking microrheology. This technique involves determining the mean squared displacement (MSD) of micrometer-sized fluorescently labeled tracer particles subject to Brownian motion within the material over time. Whereas 
A
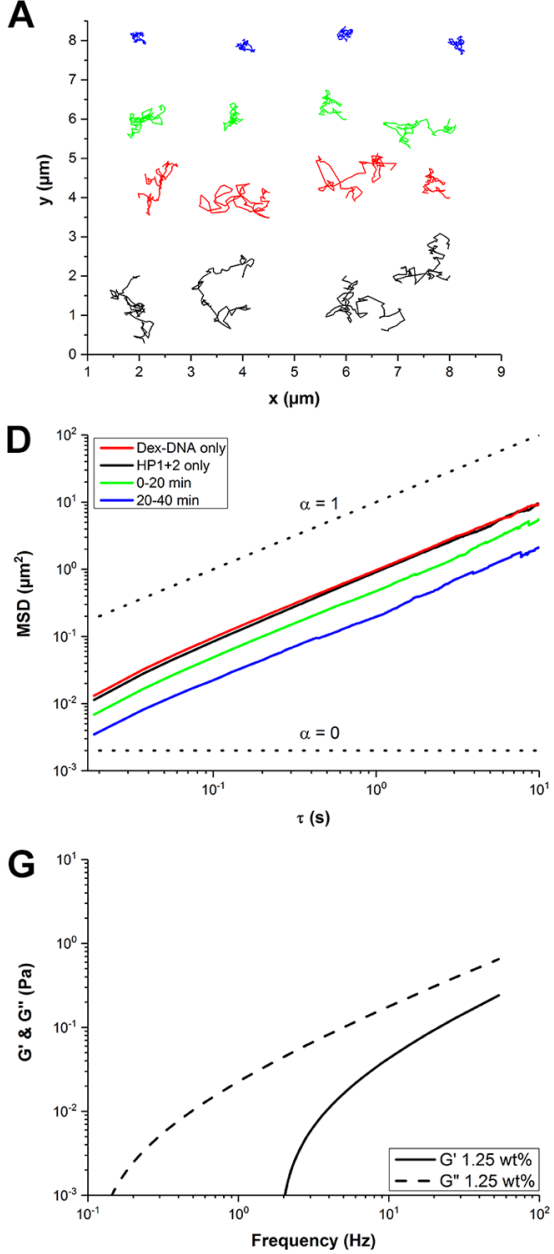

B
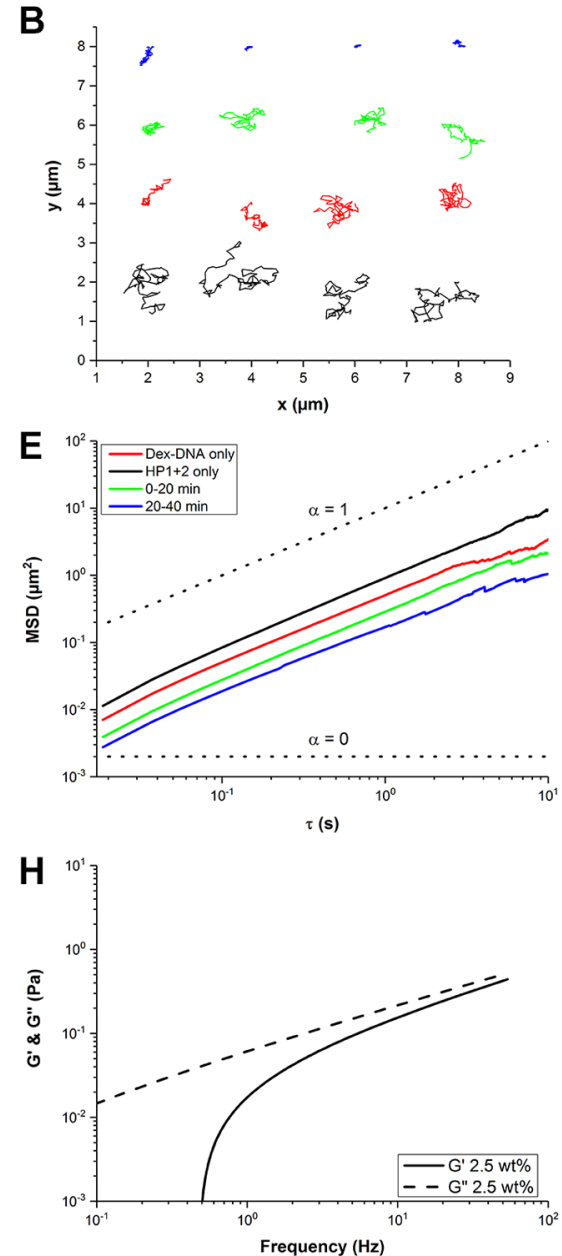
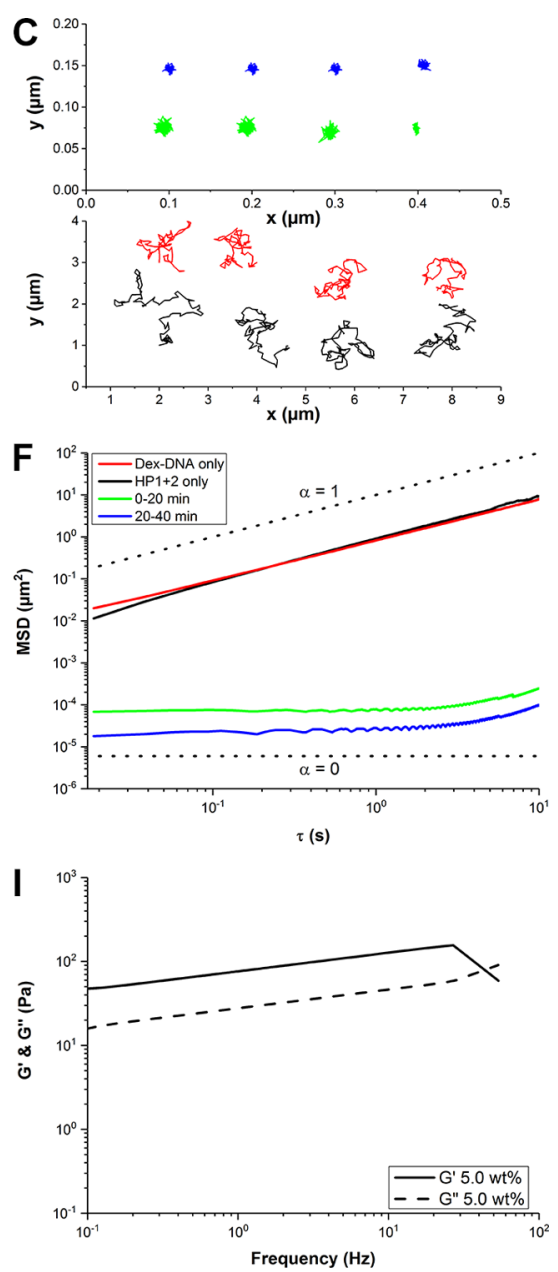

Figure 4. Particle tracking microrheology on initiator DNA-dextran graft copolymers after DNA hairpin addition (0.16 (left panel), 0.33 (middle panel) and $0.66 \mathrm{mM}$ (right panel) of initiator DNA-dextran and 0.5, 1.0 and $2.0 \mathrm{mM}$ of each DNA hairpin, respectively). Black: HP1 + HP2 only. Red: initiator DNA-dextran only. Green: HCR containing initiator DNA-dextran, HP1 and HP2 0-20 min. Blue: ibid., 20-40 min. (A, B, C) Representative collections of displacement tracks for four beads per test condition (D, E, F). MSD versus lag time plots $(G, H, I)$. Storage $\left(G^{\prime}\right)$ and loss $\left(\mathrm{G}^{\prime \prime}\right)$ moduli as a function of frequency (solid lines, $\mathrm{G}^{\prime}$; dashed lines, $\mathrm{G}^{\prime \prime}$ ).

conventional oscillatory rheology requires large sample volumes, particle-tracking microrheology requires volumes as low as $10 \mu \mathrm{L}$, which is highly advantageous for screening DNA-based materials. Fluorescently labeled polystyrene beads $1 \mu \mathrm{m}$ in diameter were mixed into solutions of: HP1 and HP2 (Figure 4 A, B, C, black tracks), the initiator DNA-dextran graft copolymer before (red tracks) and after hairpin addition (green tracks). In these experiments, concentrations of the initiator DNA-graft copolymer of $0.16,0.33$ and $0.66 \mathrm{mM}$ were examined and a 3 -fold molar amount of each HP1 and HP2 were added. For the various conditions, the bead tracks were followed over time to monitor the self-assembly process of the DNA-grafts (green tracks, 0-20 min; blue tracks, 20-40 min). The combination of the initiator DNA-dextran graft copolymer and both hairpins HP1 and HP2 showed significantly reduced Brownian motioninduced bead displacements over time in comparison to control samples. The strongest reduction of particle motion was observed for the $0.66 \mathrm{mM}$ initiator DNA-dextran graft copolymer sample, such that axes with smaller increments for $\mathrm{x}$ and $\mathrm{y}$ displacements were required for better visualization. These particle tracks were converted into MSDs and plotted with respect to time by time-wise data segmentation (Figure 4, parts $\mathrm{D}, \mathrm{E}$ and $\mathrm{F}$, respectively. Control samples containing a combination of HP1 and HP2 (black) or the initiator DNA-dextran graft copolymer only (red) displayed a linear increase in their MSDs over lag time, consistent with the behavior of Newtonian fluids for all sample concentrations. Addition of HP1 and HP2 to the initiator DNA-dextran copolymer resulted in a decrease of the MSD values with respect to time (green, 0-20 min; blue, 20-40 min) for the samples containing 0.16 or $0.33 \mathrm{mM}$ of the initiator DNA-dextran graft copolymer, indicative of increasingly viscous materials. For the sample containing $0.66 \mathrm{mM}$ of the initiator DNA-dextran graft copolymer, a decrease in both the MSD values as well as a slope of zero was observed on par with the rapid formation of a gelphase material. As a control, performing HCR on a sample consisting of $0.33 \mathrm{mM}$ of the unconjugated initiator DNA with $1.0 \mathrm{mM}$ of each DNA hairpin did not result in the formation of equally viscous materials as seen in samples containing the initiator DNA-dextran graft copolymer (Figure S6).

To gain further insight into the mechanical properties of the materials, the storage $\left(G^{\prime}\right)$ and loss $\left(G^{\prime \prime}\right)$ moduli were extracted from the complex modulus obtained from a numerical approximation of the Laplace transform of the MSD data. $G^{\prime}$ and $G^{\prime \prime}$ of the various samples $(0.16,0.33$ or $0.66 \mathrm{mM}$ initiator DNAdextran graft copolymer samples with the corresponding amount of DNA hairpins, Figure 4, parts G, H, and I, respectively) as a function of frequency were determined after $40 \mathrm{~min}$. 
As expected, for the 0.16 and $0.33 \mathrm{mM}$ initiator DNA-dextran graft copolymer samples, $G^{\prime \prime}$ was larger than $G$ ' over the entire frequency range consistent with liquid-like behavior. Conversely, for the $0.66 \mathrm{mM}$ initiator DNA-dextran graft copolymer sample $G^{\prime}$ was larger than $G^{\prime \prime}$, synonymous with the formation of a viscoelastic material. Most likely, the observed rheological behavior of the $0.66 \mathrm{mM}$ initiator DNA-graft polymer sample is as a result of surpassing the overlap concentration upon extension of the DNA grafts by the hybridization chain reaction.

\section{CONCLUSIONS}

The DNA hybridization chain reaction is a hallmark example of dynamic DNA nanotechnology that can be used for sophisticated applications in detection with limits in the femtomolar range. We have shown that this technique based on DNA strand displacement can be applied on covalent polymers to drive on-demand growth of their side chains with the potential to form macroscale materials depending on concentration through hierarchical self-assembly. In combination with the advances in DNA solid phase synthesis and its continuously decreasing production costs, we envisage that implementing this technique on polymer materials opens up this area to a whole new level of structural abstraction, allowing for the future development of a wide range of responsive materials for biomedical applications using viscosity-based changes as a readout.

\section{ASSOCIATED CONTENT}

\section{S Supporting Information}

The Supporting Information is available free of charge on the ACS Publications website at DOI: 10.1021/acs.macromol.7b02610.

Materials and methods, the synthetic scheme, and Figures S1-S6 (PDF)

\section{AUTHOR INFORMATION}

\section{Corresponding Author}

*E-mail: r.e.kieltyka@chem.leidenuniv.nl. (R.E.K.).

\section{ORCID}

Ilja K. Voets: 0000-0003-3543-4821

Roxanne E. Kieltyka: 0000-0001-9152-1810

\section{Notes}

The authors declare no competing financial interest.

\section{ACKNOWLEDGMENTS}

AFM measurements were performed in the AFM Lab of the Leiden Institute of Physics (LION). R.E.K. (NWO-ECHOSTIP Grant 717.014.005, NWO-VENI Grant 722.012.011) and I.K.V. (NWO-ECHO-STIP Grant 717.013.005, NWO-VIDI Grant 723.014.006) would like to acknowledge The Netherlands Organization for Scientific Research (NWO).

\section{REFERENCES}

(1) McLaughlin, C. K.; Hamblin, G. D.; Sleiman, H. F. Supramolecular DNA Assembly. Chem. Soc. Rev. 2011, 40 (12), 5647-5656.

(2) Seeman, N. C. DNA in a Material World. Nature 2003, 421 (6921), 427-431.

(3) Pinheiro, A. V.; Han, D.; Shih, W. M.; Yan, H. Challenges and Opportunities for Structural DNA Nanotechnology. Nat. Nanotechnol. 2011, 6 (12), 763-772.

(4) Liu, J. Oligonucleotide-Functionalized Hydrogels as Stimuli Responsive Materials and Biosensors. Soft Matter 2011, 7 (15), 6757.

(5) Rothemund, P. W. K. Folding DNA to Create Nanoscale Shapes and Patterns. Nature 2006, 440 (7082), 297-302.
(6) Sobczak, J.-P. J.; Martin, T. G.; Gerling, T.; Dietz, H. Rapid Folding of DNA into Nanoscale Shapes at Constant Temperature. Science 2012, 338 (6113), 1458-1461.

(7) Aldaye, F. A.; Palmer, A. L.; Sleiman, H. F. Assembling Materials with DNA as the Guide. Science 2008, 321 (5897), 1795-1799.

(8) Douglas, S. M.; Bachelet, I.; Church, G. M. A Logic-Gated Nanorobot for Targeted Transport of Molecular Payloads. Science 2012, 335 (6070), 831-834.

(9) Zhang, D. Y.; Seelig, G. Dynamic DNA Nanotechnology Using Strand-Displacement Reactions. Nat. Chem. 2011, 3 (2), 103-113.

(10) Chen, Y. J.; Groves, B.; Muscat, R. A.; Seelig, G. DNA Nanotechnology from the Test Tube to the Cell. Nat. Nanotechnol. 2015, 10 (9), 748-760.

(11) Freeman, R.; Stephanopoulos, N.; Álvarez, Z.; Lewis, J. A.; Sur, S.; Serrano, C. M.; Boekhoven, J.; Lee, S. S.; Stupp, S. I. Instructing Cells with Programmable Peptide DNA Hybrids. Nat. Commun. 2017, 8, 15982 .

(12) Zhang, D. Y.; Winfree, E. Control of DNA Strand Displacement Kinetics Using Toehold Exchange. J. Am. Chem. Soc. 2009, 131 (47), 17303-17314.

(13) Li, B.; Ellington, A. D.; Chen, X. Rational, Modular Adaptation of Enzyme-Free DNA Circuits to Multiple Detection Methods. Nucleic Acids Res. 2011, 39 (16), e110.

(14) Zhang, D. Y.; Turberfield, A. J.; Yurke, B.; Winfree, E. Engineering Entropy-Driven Reactions and Networks Catalyzed by DNA. Science 2007, 318 (5853), 1121-1125.

(15) Dirks, R. M.; Pierce, N. A. Triggered Amplification by Hybridization Chain Reaction. Proc. Natl. Acad. Sci. U. S. A. 2004, 101 (43), 15275-15278.

(16) Choi, H. M. T.; Beck, V. A.; Pierce, N. A. Terms of Use NextGeneration in Situ Hybridization Chain Reaction: Higher Gain, Lower Cost, Greater Durability. ACS Nano 2014, 8 (5), 4284-4294.

(17) Seelig, G.; Soloveichik, D.; Zhang, D. Y.; Winfree, E. EnzymeFree Nucleic Acid Logic Circuits. Science 2006, 314 (5805), 15851588.

(18) Shin, J.; Pierce, N. A. A Synthetic DNA Walker for Molecular Transport. J. Am. Chem. Soc. 2004, 126 (35), 10834-10835.

(19) Sherman, W. B.; Seeman, N. C. A Precisely Controlled DNA Biped Walking Device. Nano Lett. 2004, 4 (7), 1203-1207.

(20) Zhang, D. Y.; Winfree, E. Dynamic Allosteric Control of Noncovalent DNA Catalysis Reactions. J. Am. Chem. Soc. 2008, 130 (42), 13921-13926.

(21) Zhang, D. Y.; Hariadi, R. F.; Choi, H. M. T.; Winfree, E. Integrating DNA Strand-Displacement Circuitry with DNA Tile SelfAssembly. Nat. Commun. 2013, 4, 1965.

(22) Xu, Q.; Zhu, G.; Zhang, C. Y. Homogeneous Bioluminescence Detection of Biomolecules Using Target-Triggered Hybridization Chain Reaction-Mediated Ligation without Luciferase Label. Anal. Chem. 2013, 85 (14), 6915-6921.

(23) Rudchenko, M.; Taylor, S.; Pallavi, P.; Dechkovskaia, A.; Khan, S.; Butler, V. P., Jr; Rudchenko, S.; Stojanovic, M. N. Autonomous Molecular Cascades for Evaluation of Cell Surfaces. Nat. Nanotechnol. 2013, 8 (8), 580-586.

(24) Shao, Y.; Jia, H.; Cao, T.; Liu, D. Supramolecular Hydrogels Based on DNA Self-Assembly. Acc. Chem. Res. 2017, 50 (4), 659-668.

(25) Serpell, C. J.; Edwardson, T. G. W.; Chidchob, P.; Carneiro, K. M. M.; Sleiman, H. F. Precision Polymers and 3D DNA Nanostructures: Emergent Assemblies from New Parameter Space. J. Am. Chem. Soc. 2014, 136 (44), 15767-15774.

(26) Noteborn, W. E. M.; Zwagerman, D. N. H.; Talens, V. S.; Maity, C.; van der Mee, L.; Poolman, J. M.; Mytnyk, S.; van Esch, J. H.; Kros, A.; Eelkema, R.; Kieltyka, R. E. Crosslinker-Induced Effects on the Gelation Pathway of a Low Molecular Weight Hydrogel. Adv. Mater. 2017, 29 (12), 1603769.

(27) Vyborna, Y.; Vybornyi, M.; Häner, R. Functional DNA-Grafted Supramolecular Polymers - Chirality, Cargo Binding and Hierarchical Organization. Chem. Commun. 2017, 53 (37), 5179-5181. 
(28) Chen, P.; Li, C.; Liu, D.; Li, Z. DNA-Grafted Polypeptide Molecular Bottlebrush Prepared via Ring-Opening Polymerization and Click Chemistry. Macromolecules 2012, 45 (24), 9579-9584.

(29) Wilks, T. R.; Bath, J.; de Vries, J. W.; Raymond, J. E.; Herrmann, A.; Turberfield, A. J.; O'Reilly, R. K. Giant Surfactants" Created by the Fast and Efficient Functionalization of a DNA Tetrahedron with a Temperature-Responsive Polymer. ACS Nano 2013, 7 (10), 85618572 .

(30) Rodríguez-Pulido, A.; Kondrachuk, A. I.; Prusty, D. K.; Gao, J.; Loi, M. A.; Herrmann, A. Light-Triggered Sequence-Specific Cargo Release from DNA Block Copolymer-Lipid Vesicles. Angew. Chem., Int. Ed. 2013, 52 (3), 1008-1012.

(31) Soontornworajit, B.; Zhou, J.; Shaw, M. T.; Fan, T.-H.; Wang, Y. Hydrogel Functionalization with DNA Aptamers for Sustained PDGFBB Release. Chem. Commun. 2010, 46 (11), 1857.

(32) Kahn, J. S.; Trifonov, A.; Cecconello, A.; Guo, W.; Fan, C.; Willner, I. Integration of Switchable DNA-Based Hydrogels with Surfaces by the Hybridization Chain Reaction. Nano Lett. 2015, 15 (11), 7773-7778.

(33) Liao, W.-C.; Lilienthal, S.; Kahn, J.; Riutin, M.; Sohn, Y. S.; Nechushtai, R; Willner, I. PH- and Ligand-Induced Release of Loads from DNA-Acrylamide Hydrogel Microcapsules. Chem. Sci. 2017, 8, $3362-3373$.

(34) Tan, X.; Lu, X.; Jia, F.; Liu, X.; Sun, Y.; Logan, J. K.; Zhang, K. Blurring the Role of Oligonucleotides: Spherical Nucleic Acids as a Drug Delivery Vehicle. J. Am. Chem. Soc. 2016, 138 (34), 1083410837.

(35) Ohno, S.; Matyjaszewski, K. Controlling Grafting Density and Side Chain Length in Poly(n-Butyl Acrylate) by ATRP Copolymerization of Macromonomers. J. Polym. Sci., Part A: Polym. Chem. 2006, 44 (19), 5454-5467.

(36) Lee, H. Il; Pietrasik, J.; Sheiko, S. S.; Matyjaszewski, K. StimuliResponsive Molecular Brushes. Prog. Polym. Sci. 2010, 35 (1-2), 2444.

(37) Feng, C.; Li, Y.; Yang, D.; Hu, J.; Zhang, X.; Huang, X. WellDefined Graft Copolymers: From Controlled Synthesis to Multipurpose Applications. Chem. Soc. Rev. 2011, 40 (3), 1282-1295.

(38) Sheiko, S. S.; Sumerlin, B. S.; Matyjaszewski, K. Cylindrical Molecular Brushes: Synthesis, Characterization, and Properties. Prog. Polym. Sci. 2008, 33, 759-785.

(39) Petersen, H.; Fechner, P. M.; Martin, A. L.; Kunath, K.; Stolnik, S.; Roberts, C. J.; Fischer, D.; Davies, M. C.; Kissel, T. Polyethylenimine-Graft-Poly(Ethylene Glycol) Copolymers: Influence of Copolymer Block Structure on DNA Complexation and Biological Activities as Gene Delivery System. Bioconjugate Chem. 2002, 13 (4), 845-854.

(40) Schubert, U. S.; Hofmeier, H. Metallo-Supramolecular Graft Copolymers: A Novel Approach toward Polymer-Analogous Reactions. Macromol. Rapid Commun. 2002, 23 (9), 561-566.

(41) South, C. R.; Burd, C.; Weck, M. Modular and Dynamic Functionalization of Polymeric Scaffolds. Acc. Chem. Res. 2007, 40 (1), 63-74.

(42) Hammond, M. R.; Li, C.; Tsitsilianis, C.; Mezzenga, R. Hierarchical Self-Organization in Polyelectrolyte-Surfactant Complexes Based on Heteroarm Star Block Copolyampholytes. Soft Matter 2009, 5, 2371-2377.

(43) Yu, Y.; Chau, Y. One-Step "Click" Method for Generating Vinyl Sulfone Groups on Hydroxyl-Containing Water-Soluble Polymers. Biomacromolecules 2012, 13 (3), 937-942.

(44) Gao, H.; Yang, M.; Cook, A. F. Stabilization of Double-Stranded Oligonucleotides Using Backbone-Linked Disulfide Bridges. Nucleic Acids Res. 1995, 23 (2), 285-292.

(45) Chandran, H.; Rangnekar, A.; Shetty, G.; Schultes, E. A.; Reif, J. H.; Labean, T. H. An Autonomously Self-Assembling Dendritic DNA Nanostructure for Target DNA Detection. Biotechnol. J. 2013, 8 (2), 221-227.

(46) Rachofsky, E. L.; Osman, R.; Ross, J. B. Probing Structure and Dynamics of DNA with 2-Aminopurine: Effects of Local Environment on Fluorescence. Biochemistry 2001, 40 (4), 946-956.
(47) Armstrong, J. K.; Wenby, R. B.; Meiselman, H. J.; Fisher, T. C. The Hydrodynamic Radii of Macromolecules and Their Effect on Red Blood Cell Aggregation. Biophys. J. 2004, 87 (6), 4259-4270.

(48) Antoniou, E.; Tsianou, M. Solution Properties of Dextran in Water and in Formamide. J. Appl. Polym. Sci. 2012, 125 (3), 16811692.

(49) Zadeh, J. N.; Steenberg, C. D.; Bois, J. S.; Wolfe, B. R.; Pierce, M. B.; Khan, A. R.; Dirks, R. M.; Pierce, N. A. NUPACK: Analysis and Design of Nucleic Acid Systems. J. Comput. Chem. 2011, 32 (1), 170173.

(50) Foster, J. C.; Radzinski, S. C.; Matson, J. B. Graft Polymer Synthesis by RAFT Transfer-To. J. Polym. Sci., Part A: Polym. Chem. 2017, 55 (18), 2865-2876. 